\title{
Binary Representations of Fingerprint Spectral Minutiae Features
}

\author{
Haiyun Xu and Raymond N.J. Veldhuis \\ Department of Electrical Engineering, University of Twente \\ $\{h . x u, r . n . j . v e l d h u i s\} @ e w i . u t w e n t e . n l$
}

\begin{abstract}
A fixed-length binary representation of a fingerprint has the advantages of a fast operation and a small template storage. For many biometric template protection schemes, a binary string is also required as input. The spectral minutiae representation is a method to represent a minutiae set as a fixed-length real-valued feature vector. In order to be able to apply the spectral minutiae representation with a template protection scheme, we introduce two novel methods to quantize the spectral minutiae features into binary strings: Spectral Bits and Phase Bits. The experiments on the FVC2002 database show that the binary representations can even outperformed the spectral minutiae real-valued features.
\end{abstract}

\section{Introduction}

Minutiae-based matching is the most widely used technique in fingerprint recognition systems. However, the low matching speed is limiting its application. At the same time, increasing security and privacy concerns make minutiae template protection a crucial task. The spectral minutiae representation is a method to represent a minutiae set as a fixed-length feature vector, which is invariant to translation, and in which rotation and scaling become translations, so that they can be easily compensated for. These characteristics enable the combination of fingerprint recognition systems with template protection schemes and allow for a fast minutiae-based matching as well.

In order to apply the spectral minutiae representation with a template protection scheme, for example, based on a fuzzy extractor [3] or fuzzy commitment [4], we need to quantize the spectral minutiae real-valued features into binary strings. A fixed-length binary representation also has additional advantages such as small template storage and high matching speed.

The main contributions of this paper are: we propose two novel quantization schemes to convert the real- valued spectral minutiae features into binary strings: Spectral Bits and Phase Bits. We also evaluate our algorithms on the FVC2002-DB2 database and show that the two binary representations achieved very promising results.

In this paper, we will first present the complex spectral minutiae representation (SMC), a new spectral minutiae representation method in Section 2. Next, in Section 3, we will briefly review the Column-PCA and Line-DFT feature reduction algorithms, which can greatly compact the spectral minutiae features. Then, in Section 4, we will introduce two novel methods to quantize the spectral minutiae features into binary strings: Spectral Bits and Phase Bits. Finally, we will show the experimental results in Section 5 and draw conclusions in Section 6.

\section{Complex Spectral Minutiae Represen- tation}

The objective of the spectral minutiae representation is to represent a minutiae set as a fixed-length feature vector, which is invariant to translation and rotation [7, 9]. We assume that the scaling has already been compensated for on the level of the minutiae sets. This is for instance possible if minutiae are presented in a standard like [2], which includes sensor resolution. In Figure 1, the procedure of the complex spectral minutiae representation (SMC) is illustrated.

Assume a fingerprint with $Z$ minutiae. First, we code the minutiae locations by indicator functions, which are isotropic two-dimensional Gaussian functions in the spatial domain. Then we incorporate the minutiae orientation by assigning each Gaussian a complex amplitude $\mathrm{e}^{\mathrm{j} \theta_{i}}, i=1 \ldots Z$. In this way, we represent minutiae points as complex valued continuous functions, the magnitude of which is shown in Figure 1(b). In this representation, translation and rotation may exist, depending on how the user has put his finger on the sensor.

Next, a two-dimensional continuous Fourier trans- 


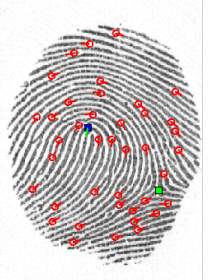

(a)

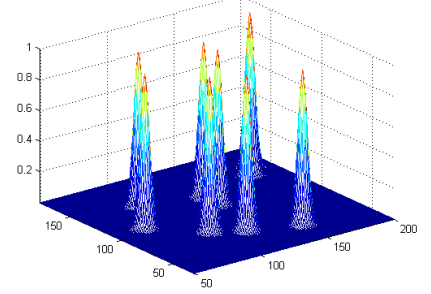

(b)

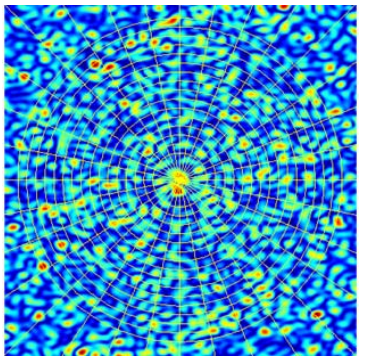

(c)

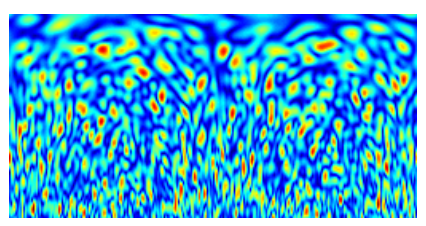

(d)

Figure 1. Illustration of the complex spectral minutiae representation procedure. (a) a fingerprint and its minutiae; (b) representation of minutiae points as complex valued continuous functions; (c) the 2D Fourier spectrum of ' $b$ ' in a Cartesian coordinate and a polar sampling grid; (d) the Fourier spectrum sampled on a polar grid.

form is performed and only the Fourier magnitude is kept, illustrated in Figure 1(c). This representation is now translation invariant according to the shift property of the continuous Fourier transform. This representation can be computed analytically,

$$
\begin{aligned}
& \mathcal{M}_{\mathrm{C}}\left(\omega_{\mathrm{x}}, \omega_{\mathrm{y}} ; \sigma_{\mathrm{C}}^{2}\right)= \\
& \left|\exp \left(-\frac{\omega_{\mathrm{x}}^{2}+\omega_{\mathrm{y}}^{2}}{2 \sigma_{\mathrm{C}}^{-2}}\right) \sum_{i=1}^{Z} \exp \left(-\mathrm{j}\left(\omega_{\mathrm{x}} x_{i}+\omega_{\mathrm{y}} y_{i}\right)+\mathrm{j} \theta_{i}\right)\right|,
\end{aligned}
$$

with $\left(x_{i}, y_{i}, \theta_{i}\right)$ the location and orientation of the $i$-th minutia in the fingerprint image, and $\left(\omega_{\mathrm{x}}, \omega_{\mathrm{y}} ; \sigma_{\mathrm{C}}^{2}\right)$ are the spatial frequencies and the Gaussian parameter respectively.

Finally, the Fourier spectrum is re-mapped onto a polar coordinate system, illustrated in Figure 1(d). In the radial direction $\lambda$, we use $M=128$ samples between $\lambda_{\mathrm{l}}=0.05$ and $\lambda_{\mathrm{h}}=0.58$. In the angular direction $\beta$, we use $N=256$ samples uniformly distributed between $\beta=0$ and $\beta=2 \pi$. Since our target application is in a high security scenario with reasonable good quality fingerprints, we choose $\sigma_{\mathrm{C}}=0$ for the best performance. In this case, there is no multiplication with Gaussian in the frequency domain. An analysis of the selection of the Gaussian parameter $\sigma$ can be found in [7]. According to the rotation properties of the two-dimensional continuous Fourier transform, now the rotation becomes translation along the new coordinate axis.

Let $R(m, n)$ and $T(m, n)$ be the two sampled minutiae spectra that are achieved from the reference fingerprint and test fingerprint respectively. Both $R(m, n)$ and $T(m, n)$ are normalized to have zero mean and unit energy. We use the two-dimensional correlation coefficient between $R$ and $T$ as a measure of their similarity.

In practice, the input fingerprint images are rotated. Therefore, we need to test a few rotations, which become the circular shifts in the horizontal direction. We denote $T(m, n-j)$ as a circularly shifted version of $T(m, n)$ and the final matching score between $R$ and $T$ is,

$$
\begin{array}{r}
S^{(R, T)}=\max _{j}\left\{\frac{1}{M N} \sum_{m, n} R(m, n) T(m, n-j)\right\} \\
-15 \leq j \leq 15 .
\end{array}
$$

\section{Feature Reduction}

The spectral minutiae feature is a 32,768dimensional real-valued feature vector. The large dimensionality of the spectral minutiae feature can cause three problems. First, the template storage requirement is very high. Second, the high dimensionality leads to a computational burden and the matching speed will be limited. Third, the high dimensionality can lead to a small sample size problem [6]. In order to cope with these problems, we will apply two feature reduction methods that are introduced in [8]: the Column Principal Component Analysis (CPCA) and the Line Discrete Fourier Transform (LDFT) feature reduction algorithms, which can be applied in conjunction.

The idea of Column PCA (CPCA) is applying the well known Principal Component Analysis technique to the SMC columns. PCA has two functions: it decorrelates features and concentrates power. The CPCA representation is shown in Figure 2(b). We can see that after CPCA, the power is concentrated in the upper lines. 


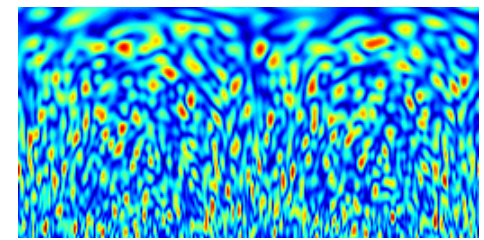

(a)

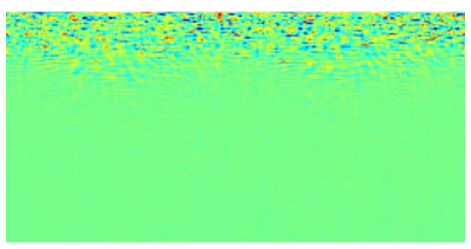

(b)

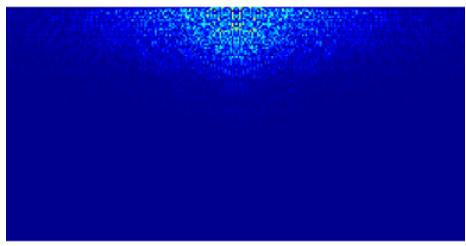

(c)

Figure 2. Illustration of the CPCA transform and the LDFT representation. (a) the SMC feature; (b) the minutiae spectrum after the CPCA transform; (c) the magnitude of the LDFT representation of (b).

The features in the lower parts are close to zero, so we can remove them from the representation.

After the CPCA feature reduction, the minutiae spectrum remains periodic on the horizontal axis. Therefore, applying Discrete Fourier Transform to each line generates an exact representation. From Figure 2(c), we can see that the Fourier coefficients concentrate power in the low frequency part. Therefore, we can achieve feature reduction by removing the Fourier coefficients in the higher frequency part. In this paper, we improved the LDFT feature reduction algorithm represented in [8]. In our previous work [8], we kept the energy in each line of the spectral minutiae feature constant when reducing the Fourier coefficients. However, since the energy of each line is probably unevenly distributed, see Figure 2(c), this may not be the most efficient way to perform feature reduction. In this paper, we keep a fixedlength Fourier coefficients with a maximum overall energy retainment. We gain this information in the training procedure. In this way, we can increase the feature reduction rate from $51 \%$ to $78 \%$ while keeping a comparable performance (we will show the details in Section 5).

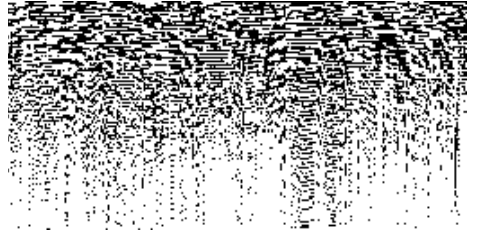

(a)

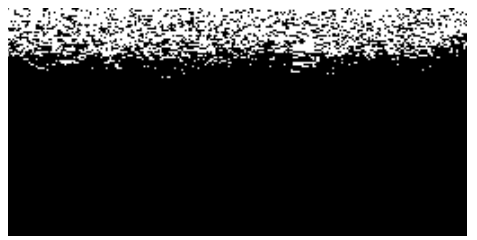

(b)

Figure 3. Example of Spectral Bits (SMC spectra after CPCA). (a) the Sign Bit; (b) the Mask Bit.

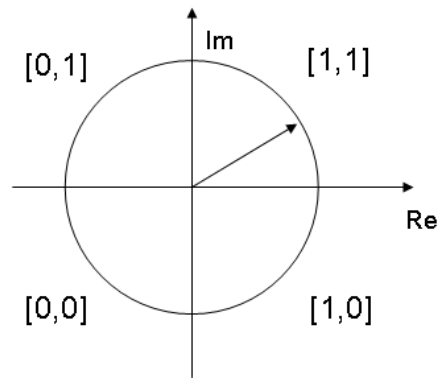

Figure 4. Quantization scheme.

\section{Quantization}

In this section, we will introduce two quantization methods: Spectral Bits, which is applied in conjunction with the CPCA feature reduction, and Phase Bits, which is applied in conjunction with the LDFT feature reduction.

\subsection{Spectral Bits}

The Spectral Bits quantization is applied to the realvalued features after the CPCA feature reduction. First, each real-valued feature is quantized as one bit ( 1 if the feature is greater than zero and 0 otherwise, we call it sign bit), shown in Figure 3(a). Second, since the quantization boundary is zero, and the features close to zero are unstable and likely to flip, they may cause errors. Therefore, we will mask out the features of which the absolute values are below a certain threshold. For the best recognition performance, we set the threshold to 0.8 after normalizing the spectra to have unit energy. 


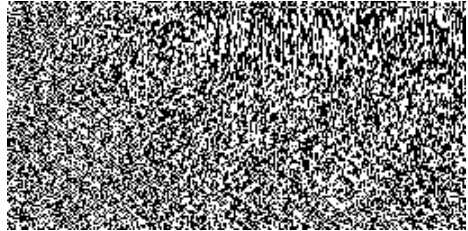

(a)

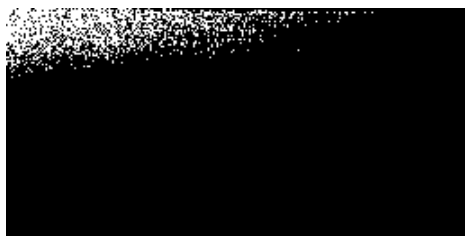

(b)

Figure 5. Example of Phase Bits (SMC spectra after LDFT). (a) the Sign Bit; (b) the Mask Bit.

We found this parameter empirically by testing thresholds on different fingerprint databases. The resulting mask bit is shown in Figure 3(b).

\subsection{Phase Bits}

The Phase Bits quantization is applied to the complex-valued features after the LDFT feature reduction. Each complex component is quantized as 2 bits and the quantization scheme is shown in Figure 4. The sign bit and the mask bit generation is similar as the procedure in the Spectral Bits quantization, shown in Figure 5. We set the mask threshold to 1.2 empirically after normalizing the spectra to have unit energy.

\subsection{Fractional Hamming Distance}

After generating sign bit and mask bit vectors, we can compute a fractional Hamming distance (HD) as a measure of the dissimilarity between two fingerprints spectra $R(m, n)$ and $T(m, n)$, whose sign bit vectors are denoted as $\{$ codeR, code $\mathrm{T}\}$ and mask bit vectors as $\{$ maskR, maskT $\}$,

$$
H D=\frac{\|(\operatorname{codeR} \otimes \operatorname{code} \mathrm{T}) \cap \operatorname{maskR} \cap \operatorname{maskT}\|}{\|\operatorname{maskR} \cap \operatorname{mask} \mathrm{T}\|} .
$$

\section{Results}

The proposed algorithms have been evaluated on the FVC2002-DB2 [5] fingerprint database. We apply the
Table 1. Results.

\begin{tabular}{|l|c|c|}
\hline Methods & EER & FRR @ FAR0.1\% \\
\hline \hline 1. Real features & $3.2 \%$ & $6.1 \%$ \\
\hline 2. Spectral Bits & $3.2 \%$ & $5.9 \%$ \\
\hline 3. Phase Bits & $3.8 \%$ & $6.3 \%$ \\
\hline 4. Fusion of (2) and (3) & $3.0 \%$ & $5.4 \%$ \\
\hline
\end{tabular}

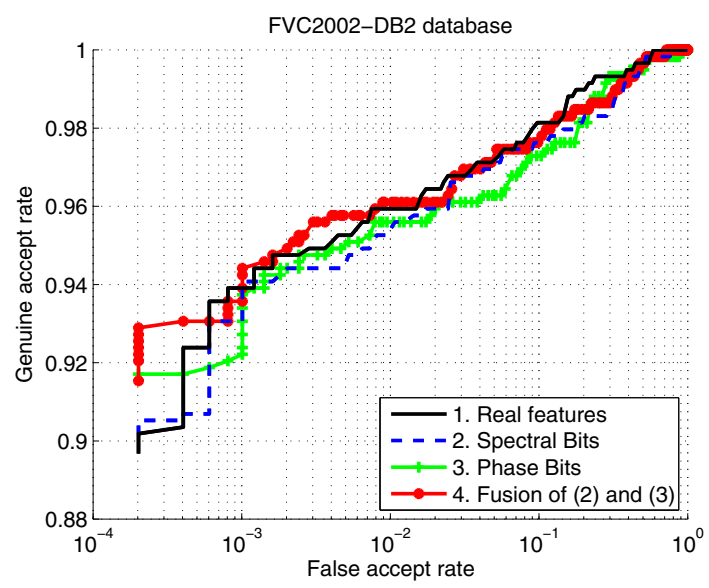

Figure 6. ROC curves.

same experimental protocol as in the FVC competition: the samples from finger ID 101 to 110 for the CPCA and LDFT training and samples from person ID 1 to 100 for test. We propose to use our algorithm in a high security scenario. In the FVC2002-DB2 databases, samples 3, 4, 5 and 6 were obtained by requesting the users to provide fingerprints with exaggerated displacement and rotation. In a high security scenario where the user is aware that cooperation is crucial for security reasons, he will be cooperative. Therefore, we chose samples 1, 2, 7 and 8 for a more realistic evaluation. To deal with the large rotations, an absolute pre-alignment based on core and its direction can be applied. We obtain the minutiae sets using the VeriFinger minutiae extractor [1](VeriFinger Extractor Version 5.0.2.0 is used).

We test our algorithm in a verification setting. For matching genuine pairs, we used all the possible combinations. For matching imposter pairs, we chose the first sample from each identity. Therefore, we have totally 600 genuine scores and 4950 imposter scores.

In our experiment, we also use the core as a reference point to assist the verification, following the procedure in [7] (for each fingerprint, maximum two cores or/and two deltas were used to improve the performance in [7], while in this paper, only the upper core is used).

We test both the Spectral Bits and the Phase Bits results. For the CPCA feature reduction, we keep the top 35 lines, with a feature reduction rate of $73 \%$. There- 
fore, we generate 8960 bits for the Spectral Bits string, among which we mask out about $40 \%$. For the LDFT feature reduction, we keep the first 1000 components with largest magnitudes, with a feature reduction rate of $78 \%$. Thus, we generate 2000 bits for the Phase Bits string, among which we mask out about $45 \%$. For the LDFT feature reduction, it should be noted that if we apply the LDFT feature reduction on SMC features using the method in [8], we can only achieve a feature reduction rate of $51 \%$ for the comparable performance.

For comparison, we also present the results of realvalued features (before quantization). To improve the binary results, we also tried the fusion of the Spectral Bits and the Phase Bits (a score level sum-rule fusion is applied). The results are shown in Tables 1 and the ROC curves are shown in Figures 6. From the results, we can see that both the Spectral Bits and the Phase Bits hardly degrade the performance compared with the real-valued features. The Spectral Bits performs better than the Phase Bits. The fusion of these two quantization schemes gives the best performance, which is also outperformed the spectral minutiae real-valued features.

\section{Discussion and Conclusion}

In this paper, we introduce two methods to extract bits from the spectral minutiae features: the Spectral Bits and the Phase Bits. Both quantization schemes show promising results. These two methods are based on the spectral minutiae features after the CPCA and LDFT feature reduction, which has already decreased the computational complexity for more than 10 times [8]. The fixed-length binary representations proposed in this paper further compact the template and increase the matching speed.

To apply the spectral minutiae representation with a template protection scheme, for example, based on fuzzy commitment [4], an error correction step is needed. Erasure decoding can handle the masking of bits. Furthermore, to enhance the recognition accuracy, we can use multiple enrollment to improve the biometric template quality. Investigating the possible error correction codes and multiple enrollment schemes will be our future work.

\section{Acknowlegements}

This research is supported by the ProBiTe project funding by Sentinels and the TURBINE project funding by the European Union under the Seventh Framework Programme.

\section{References}

[1] VeriFinger SDK. http: //www . neurotechnologija.com/.

[2] ISO/IEC 19794-2, Information Technology - Biometric Data Interchange Format - Part 2: Finger Minutiae Data. 2005.

[3] Y. Dodis, L. Reyzin, and A. Smith. Fuzzy extractors. In P. Tuyls, B. Skoric, and T. Kevenaar, editors, Security with Noisy Data - On Private Biometrics, Secure Key Storage and Anti-Counterfeiting, chapter 3, pages 45-56. Springer, 2007.

[4] A. Juels. Fuzzy commitment. In P. Tuyls, B. Skoric, and T. Kevenaar, editors, Security with Noisy Data - On Private Biometrics, Secure Key Storage and AntiCounterfeiting, chapter 3, pages 45-56. Springer, 2007.

[5] D. Maio, D. Maltoni, R. Cappelli, J. Wayman, and A. Jain. FVC2002: Second fingerprint verification competition. 3:811-814, Aug. 2002.

[6] S. Raudys and A. Jain. Small sample size effects in statistical pattern recognition: Recommendations for practitioners. 13(3):252-264, March 1991.

[7] H. Xu, R. Veldhuis, A. Bazen, T. Kevenaar, T. Akkermans, and B. Gokberk. Fingerprint verification using spectral minutiae representations. Information Forensics and Security, IEEE Transactions on, 4(3):397-409, Sept. 2009.

[8] H. Xu, R. Veldhuis, T. Kevenaar, and T. Akkermans. A fast minutiae-based fingerprint recognition system. Systems Journal, IEEE, 3(4):418 -427, dec. 2009.

[9] H. Xu and R. N. J. Veldhuis. Complex spectral minutiae representation for fingerprint recognition. In Proceedings of the IEEE Computer Society Conference on Computer Vision and Pattern Recognition - Workshop on Biometrics, San Francisco, USA, 2010. To appear. 